\title{
A Study of Campus Crisis From the Perspective of Social Combustion Theory
}

\author{
Xingwang Liu ${ }^{1, *}$

\begin{abstract}
${ }^{1}$ School of Economics, Guizhou University of Commerce, Guiyang, Guizhou 550014, China
*Corresponding author. Email: 201510055@gzcc.edu.cn
\end{abstract}

\begin{abstract}
Campus crisis is an eternal topic of campus management. From the perspective of social combustion theory, discord and disharmony of relationships within nature, between humans and nature, and among humans are the combustion substances that cause campus crisis. Asymmetric social public opinion and unbalanced social psychology are the combustion promoters of the campus crisis. Unexpected events with a certain scale and influence are the ignition temperature of the campus crisis. The interaction of combustion substance, combustion promoter and ignition temperature leads to campus crisis. The effective strategies to address the campus crisis are to manage the combustion substance, to control the combustion promoter and to prevent the ignition temperature from being too high.
\end{abstract}

Keywords: campus crisis, cause of crisis, social combustion theory, crisis management

\section{EMERGENCE OF THE ISSUE}

In recent years, the occurrence of campus crisis in China sees trends of great numbers, high in frequency, and simultaneity, which engenders increasingly serious loses in a wider range and becomes more difficult to manage. Campus crisis has become a major problem confusing Chinese educators at present. The reason that the campus crisis is hard to manage lies in both the diversity and particularity of stakeholders and the complexity of its causal mechanism. Generally speaking, the campus crisis is the result of deteriorating diverse systems, namely nature, society, humanity, psychology, science and technology. Therefore, to analyze the cause of the campus crisis, it is necessary to include theory outcomes about disaster study in social science and to learn and adopt related research achievements in natural science.[1] Social physics, inbetween nature science and social science, especially its social combustion theory, provides an important theoretical perspective for better reviewing and understanding the cause of campus crisis.[2]

Social combustion theory is one of the three theories in social physics.[3] It is originally put forward by Prof. Wenyuan Niu of the Chinese Academy of Sciences, who compares crisis events in society with combustion phenomena in nature. The occurrence, development and evolution mechanism of social crisis are the results of the interaction of three basic elements which are combustion substance, combustion promoter, and ignition temperature.[4] "combustion substance" is the source and impetus of crisis, "combustion promoter" the process and catalyst, and "ignition temperature" the blasting fuse and trigger threshold.[5]

\section{CAMPUS CRISIS FROM THE PERSPECTIVE OF SOCIAL COMBUSTION THEORY}

\section{1. "Combustion substance" in the campus crisis}

In philosophy, crisis, about anthropocentrism, is the unity of human history sphere and natural eternity sphere and possesses both natural objective inevitability and social active practicality. Essentially speaking, "human is part of nature", while "human" is different from "nature" which can be divided into "true nature" and "nature with human involvement".[6] The causes of all crisis (conflict) thus could be summarized as the disharmony of relationships "between human and nature", "within nature", and "among humans". "combustion substance" is the material carrier, internal cause and source of campus crisis generating mechanism. It presents a trait of spatial and temporal disparities and is influenced by both social factors, i. e. political, economics, society, culture, nation, religion, age and gender, and natural factors which are atmosphere, water, soil, noise, waste, resource and energy, etc.

- From the perspective of relationship "within nature", the "combustion substance" of campus crisis is caused by various original extreme natural disasters, for example, earthquake, flood, landslide, debris flow, hail, freeze, hurricane, thunder and lightning, heatwave and tsunami, which are triggered by mechanism discord in the material transformation and energy transferring of basic factors of "true nature" or "nature with human involvement". To improve the abilities to understand the cause of campus crisis and preventing and 
controlling crisis, it is suggested to conduct research, simulation and supervision on the operation mechanism of macro and microsystems of earth layers, like lithosphere, water body, and atmosphere, from a natural science perspective.

- From the angle of the relationship "between human and nature", the cause of "combustion substance" of campus crisis is, from an objective aspect, the limitation of human cognition in level, width, and depth, and misconduct of conscious activity from the subjective point of view, which leads to cognition limitation, technical defect, improper attitude, physical and psychological discomfort, imbalance of practice structure, and insufficient management institution. As a result, natural disasters, like a landslide, debris flow, acid rain, haze, sand storm, and accident disasters, such as food hygiene safety, engineering construction disaster, equipment and facility failure, environment pollution, unexpected infectious disease, ecological destruction, data safety, transportation accident, fire disaster, electronic shock accident, and drowning accident, take place. It is suggested, from the perspective of engineering science, to adopt some engineering and technical methods and build necessary protective facilities and tools aiming at uplifting the prevention and control ability of campus crisis management.

To see from the relationship "among humans", "combustion substance" of campus crisis is the result of disharmony of psychological imbalance and act conflict among humans caused by factors of economics, politics, society, culture, religion, ethnic relation. For example, the poor performance of three social system mechanisms, i.e. government, market, and society, leads to the public crisis of governance failure, market disorder, and social misconduct. Conflicts among campus organization systems including party-masses departments, administrative departments, logistic departments trigger poor performance of campus institutions, and mass disturbance, like teachers' strikes and students' boycotting classes. Cognitive bias, psychological imbalance, and behavior misconduct of stakeholders, i.e. teacher, student, and management staff, in campus individual system causes individual crises such as discipline conflict, campus theft, campus drug trafficking and drug use, campus violence, administrative corruption, academic misconduct of teacher and student. It is suggested to analyze mechanisms of individual psychology, physiology and behavior and running mechanism of organization and social system from aspects of humanity and social science to build a three-level, individual-organization-society, prevention, and control mechanism of campus crisis management.

\section{2. “Combustion promoter” in campus crisis}

"Combustion promoter" in campus crisis is the element that catalyzes the entropy increase of "combustion substance" and boosts its quantitative change. It stems from stakeholder's blind following, horror, and "ignorance" caused by the uncertainty, emergency, insufficient information of crisis. Its action mechanism is closely related to factors of stakeholder, such as cognitive level, physical and psychological endurance, media's influence,[7] individual awareness of right-protection, empathy, ability to identify crisis, degree of knowledge and technical ability on disaster-response, methods and channels of disseminating information, etc. It is also influenced by stakeholders' physical social relations, namely blood relation, geographic relation, studying relation, working relation, marriage, romantic relation and right-responsibility relation, as well as all kinds of network interaction and exchange platforms of cyber society which are built on traditional or modern information technology, such as readers of e-journal and newspaper, the audience of television and broadcast, users of Wechat, QQ, Weibo, BBS and post-bar, TikTok.

\section{3. "Ignition temperature" in the campus crisis}

"Ignition temperature" means unexpected events of a certain scale and influence. It is a blasting fuse that causes campus crisis, and a fast variable that sharpens the qualitative change of entropy increase of campus "combustion substance", pushes the degree of disharmony in campus (social trauma endurance) to the threshold, and finally leads to the emergence of the campus crisis. There are several types of unexpected events that cause the campus crisis, for example:

- Political emergency in campus caused by grave educational corruption, lose of teachers' prestige, lack of channels for stakeholders to involve in society and to express interests, the impure motive of participation, poorly and flabbily organized basic party organization, weak impact of ideological and political education on students, false decision made by party-masses and administrative departments, power conflict among the right of study, right of academic, and right of the management.

- Economic emergency in campus arising from social conflicts of unfair distribution of income, such as a severe gap of wealth among teachers, students and management, improper policies and implementation about awards, praise and promotion.

- Cultural emergency in the campus as a result of teacher and student's relatively frustrated feeling and the growing sense of deprivation due to their cognitive bias, mental crisis, immoral attitude, failure of meeting individual expectation, the conflict between mainstream ideology and diverse cultures, backward concept of education, decreasing inclusiveness of culture.

- Social emergency in campus rooted in a declining degree of mutual trust among stakeholders, sharpening friction and conflict, solidifying class interest of campus, the grave gap among classes in campus, decreasing cohesive force, the unstable mood of 
teacher and student, failure of progress of teacher and student, the intervention of social issues in the campus.

- Management emergency in campus caused by lack of sound and normative management mechanism and institution of education, teaching, scientific research, poor operation and misconduct of mechanism, insufficient maintenance, and management of facility and equipment.

- Natural disaster emergency in campus triggered by an unexpected change of natural condition and campus environment, environment pollution by improper conduct of human, ecological environment destruction.

\subsection{Analysis of several "combustion phenomena" in the campus crisis}

Similar to "combustion phenomena" in nature, campus crisis could be divided into overt crisis (overt combustion) and convert crisis (convert combustion), and also be classified as a spontaneous crisis (spontaneous combustion), intermittent crisis (flash combustion) and predicable crisis (ignited combustion).

- Overt campus crisis is obvious in its trigger mechanism, that is, easily identified "combustion substance", obvious cause of the crisis, easily controlled "combustion promoter", obvious "ignition temperature". For example, architectural disaster, equipment failure, the disaster of thunder and lightning.

- Convert campus crisis is characterized as deeply hidden "combustion substance", extremely complicated cause, identification and governance requiring professional knowledge, skill and equipment as well as much time, effort and expense, various types of "combustion promoter", uncertain and uncontrollable "ignition temperature", unexpected result and impact. For example, a psychological crisis, earthquake disaster, terrorist attack, school bullying, etc.

- Spontaneous campus crisis is caused by discord relationship "within nature", namely non-human factor beyond human interaction. Its "combustion substance", "combustion promoter", and "ignition temperature" seem not to exist but are in fact beyond common cognition and difficult to perceive. For example, natural disasters like earthquakes and volcanoes, and a series of epidemic diseases caused by new viruses.
- Intermittent campus crisis features intermittent occurrence, that is, when action is ready to be taken to deal with its seeming outbreak, it disappears (false appearance in fact), but quickly reemerges once the action is called off. It is increasingly difficult to control the crisis which as a result causes growing harm. For example, intermittent natural disasters like earthquakes, volcano, tsunami, intermittent psychological disease, intermittent accident disaster caused by poor maintenance of facility and equipment, intermittent management crisis triggered by unsound institution and mechanism.

- Predictable campus crisis is characterized that the cause of "combustion substance" is obvious to detect, and "combustion promoter" and "ignition temperature" are predictable and controllable provided with certain knowledge and skill. For example, food safety, campus theft, and academic misconduct of teachers and students.

\section{THE FORMATION MECHANISM OF CAMPUS CRISIS FROM PERSPECTIVE OF SOCIAL COMBUSTION THEORY}

Campus, originally in an ordered state, gradually reveals its disharmonious side when stricken by both internal and external conflicts, such as "combustion substance" (disharmony of relations within nature, between human and nature, and among humans) accumulating, the relationship between campus systematic factors and system itself showing discord. The ordered campus thereby becomes disordered. Promoted by "combustion promoter", such as misleading information from the media, factors and relations in campus entropy increase and approximate threshold of stakeholders' social trauma endurance. Accidentally intensified by "ignition temperature" (also called campus emergency) such as political emergency, economic emergency, cultural emergency, social emergency, management emergency, campus entropy increase breaks through the threshold. As a result, the campus becomes disordered and crisis breaks out. (see Fig. 1) 


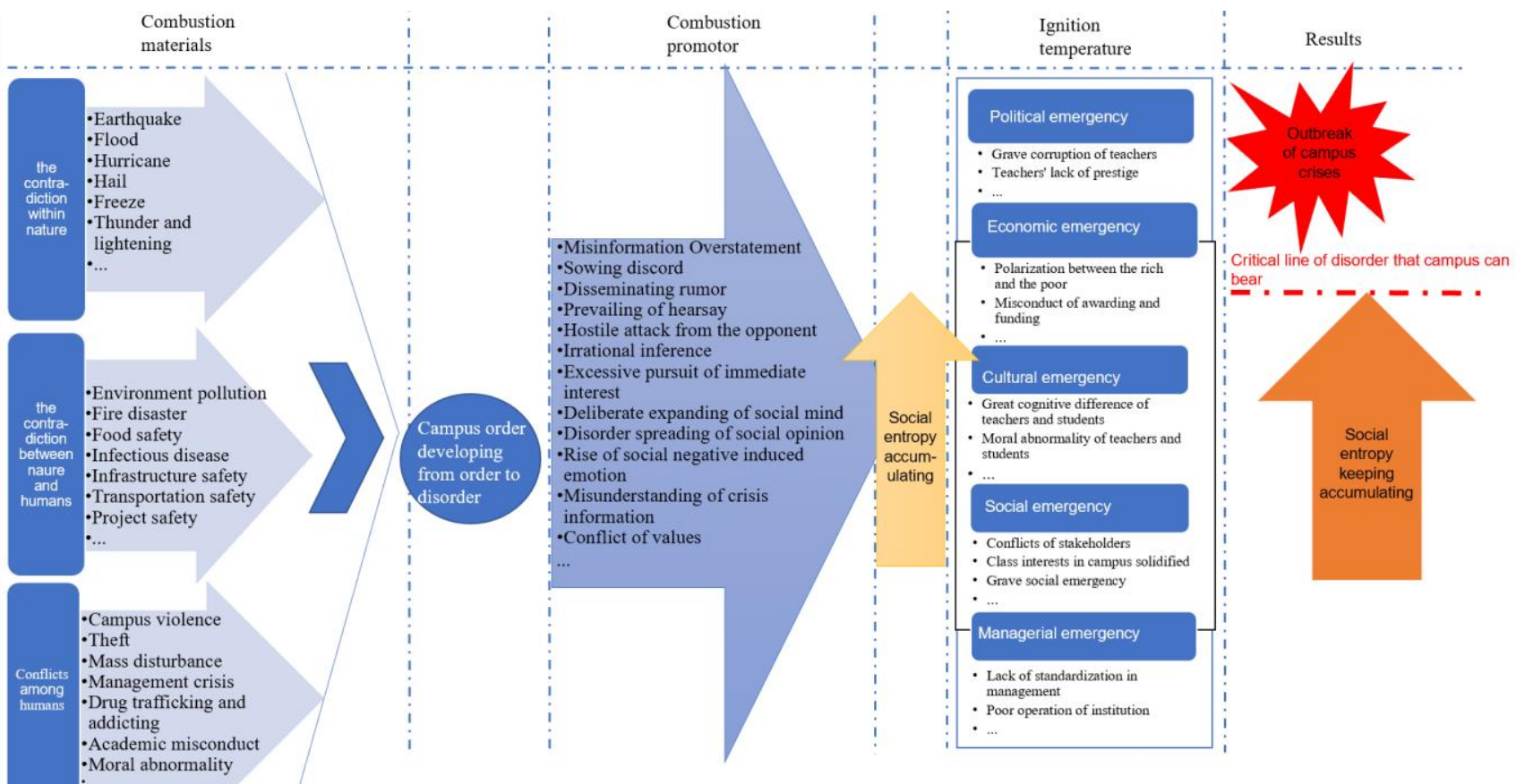

Figure 1. The formation model of the campus crisis.

\section{THE MANAGEMENT STRATEGY OF CAMPUS CRISIS FROM PERSPECTIVE OF SOCIAL COMBUSTION THEORY}

Fundamentally speaking, the campus crisis is resulted from disharmonious relations "between human and nature", "among humans", "within nature". The effective methods to stifle crisis in the cradle, to delay its occurrence, and to eliminate its negative influence including early identification and timely action to manage "combustion substance", decreasing catalytic action of "combustion promoter", strict control of "ignition temperature", enhancing the construction of facility and equipment to "prevent fire", containing entropy increase of "combustion substance" below social excitation threshold, reducing stakeholder's susceptibility to disaster, and increasing threshold value of social excitation threshold.

\subsection{Supervising "combustion substance"}

The key to the success of campus crisis governance is to identify "combustion substance", and to classify and manage "combustion substance" according to their features. "Combustion substance" in campus crisis is rich in forms, complicated informing reasons, uneven in spatial and temporal distribution, diverse. Thus, the unexpected result of misconducting "combustion substance" leads to great difficulty in managing campus crisis.

- By making full use of technology, like big data, cloud computing, artificial intelligence, internet of things, remote sensing satellite, information block, it is suggested to set up a management mechanism of campus crisis combustion substance in which "multiple domains" polymerizes, such as individual (body, psychology, cognition), society, object, technology, network, management, and diverse stakeholders collaborate. It is also beneficial to enhance the connection of problem management, risk management, disaster management, crisis management, and emergency management of campus, and to advance the digitized, automated and intelligent reform of traditional early warning mechanism of campus crisis, to make "five prevention system" (human prevention, object prevention, technological prevention, psychological prevention, and management prevention) of campus crisis "combustion substance" more scientific, more accurate, more collaborative, and more timely in identification capacity.

- It is necessary to consider all causes of campus crisis, especially features of its form, method, scope, and result, stakeholder's characteristics, spatial and temporal situation and other factors, to build and improve classification management mechanism and institution of "combustion substance" which conforms to the formation logic of campus crisis and evolving mechanism of crisis, and meets the reform need of crisis management mechanism.

-Eliminate the traditional crisis management system which is divided according to function and power. Instead, build a digital governance model of coupling structure and shared resources with the collaboration of diverse participants including chain 
of the interpersonal relationship of micro individual stakeholders (student, teacher and management), the chain of the business relationship of medium organization stakeholders (departments of partymasses, administration, logistic), and macro social stakeholders (government, market, and social organization)

\subsection{Controlling “combustion promoter"}

"Combustion promoter" of campus crisis refers to factors that booster "combustion substance" entropy increase in campus crisis. For example, the media's misleading information, overstatement, sowing discord, disseminating rumor, prevailing of hearsay, hostile attack from the opponent, irrational inference, excessive pursuit of immediate interest, deliberate expanding of the social mind,[8] disorder spreading of social opinion, the rise of social negative induced emotion, misunderstanding of crisis information, conflict of values. With the help of chain of interpersonal relationship, the chain of information dissemination network, and chain of crisis change mechanism, "combustion promoter" takes stakeholders as its core and extends from the center to marginal stakeholders and potential stakeholders in a concentric circle, which as a result knocks down stakeholders' psychological and physical front and promotes social entropy increase.

To improve the communication and collaboration mechanism of stakeholders in campus crisis, it is suggested to depend on modern technology, give play to the mechanism of government, business, social organization, market, and society, and enhance management of all participants relation chain. To uplift social system stability, it is adaptable to strengthen crisis education, to enhance crisis skill training, provide channels for expressing interest, ease stakeholders' tension, give play to unofficial opinion leader, amend the flaw of crisis management mechanism, enhance stakeholders' ability of sensing and enduring risk, and lower the inflammability of the social system.

\subsection{Preventing "ignition temperature" from being too high}

Generally speaking, "ignition temperature" (unexpected events) alone will not trigger campus crisis, and only with continuing entropy increase (i. e. increasing conflict) of "combustion substance" promoted by "combustion promoter" to approximate and break down stakeholders' physical and psychological endurance does campus crisis break out. Therefore, to decrease "ignition temperature" of campus crisis, it is suggested to restrain entropy increase of "combustion substance", and to increase the enduring ability of campus crisis stakeholders.

- To well handle the relation between "combustion substance" and "combustion promoter", it is proposed to consider both the occurrence, development, change mechanism of "combustion substance" and the function of "combustion promoter", which helps to lower speed, scale and intensity of entropy increase of campus crisis "combustion substance", stifles the crisis in the cradle, delays its occurrence and eliminates its negative influence.

- With the technology of big data, artificial intelligence, cloud computing, considering stakeholder's' factors, such as age, gender, education degree, life experience, social relationship, religious faith, economic status, working experience, family background, mental and physical status, it is necessary to set up and improve evaluation model and early warning mechanism of crisis adapting to stakeholders according to classification and grading, such as physical endurance model, psychological endurance model, economic endurance model, social adaptive capacity model, value acceptability model, social relationship harmony model. To enhance the enduring ability of campus crisis stakeholders, it is also suggested to closely supervise dynamic change of different stakeholders' endurance, cooperate with related departments and organizations to enhance intervention to stakeholders' endurance based on their reaction to different circumstances and conditions.

\section{CONCLUSION}

It is various forms of crises that exert a negative impact on the efficiency of campus crisis management. The cause of the campus crisis is essentially the discord of functions and relations among humans, between humans and nature, and within nature. This paper analyzes its cause, form and formation mechanism based on the framework of social combustion theory, and points out universal thoughts and methods of campus crisis management. The deficiency of this paper is the lack of demonstration and verification, which will become the main study focus in the future.

\section{ACKNOWLEDGMENT}

This paper is in soft science joint fund project of Guizhou Provincial Department of Science and Technology "A Study of Collaborative University Campus Crisis Management Mechanism in Guizhou-Taking Guiyang as an Example" No. LKS2010 (2014), and in natural science youth talent plan project of Guizhou Provincial Department of Education "A Study of Multi-participants Collaborative Management of Campus Crisis", No. 239 (2016), 2016 Guizhou Provincial "first-class university" construction project-special major construction project of international business (SJ-YLZY-2016-003), 2018 Guizhou University of Commerce first-class project--"top-ranking teaching 
team of international business"(project no. 2018YYLSZ02).

\section{REFERENCES}

[1] X. Tong and H. Zhang, "An analysis framework of disaster management in the context of China," Chinese Social Science, 2010, vol. 01, pp. 132-146+223-224.

[2] Y. Liu, et al. Social Physics: Social Governance, Beijing: Science Press, 2014, pp. 124.

[3] Y. Liu and W. Niu, "A study of formation and change of public opinion based on social physics," Chinese Emergency Management, 2008, vol.03, pp. 28 32.

[4] W. Liu, "The social physics and the Warning System of China's Social Stability," Bulletin of Chinese Academy of Sciences, 2001, vol. 01, pp. 15-20.

[5] X. Sun, "An analysis of optimizing methods of social combustion theory and urban safety," Science of Leader, 2018, vol 32, pp. 4-6.
[6] Editing group of Study of Marxism Fundamentals, Study of Marxism Fundamentals. Beijing: Higher Education Press, 2015, pp. 26-27.

[7] J. Ren and J. Yuan, "Related concepts and theoretical analysis of emergency management mechanism of urban public crisis events," Journal of North China University of Science and Technology, 2016, vol.16(06), pp.22-26.

[8] F. Shan and J. Gao, "Social physical interpretation of reasons for mass disturbance: the introduction of social combustion theory," Journal of Shanghai University of Finance and Economics, 2010, vol.12(06), pp.26-33.

[9] Y. Fu, Y. Liu and W. Niu, "An analysis of evolution process of mass disturbance in the construction of harmonious society," Harmonious development and systematic project - paper collection of the 15th annual conference of Systems Engineering Society of China, 2008, pp.37-43. 